Check for updates

Cite this: RSC Adv., 2019, 9, 4948

\title{
Manipulating thermal resistance at the solid-fluid interface through monolayer deposition
}

\author{
Mohammad Rashedul Hasan, ${ }^{2}$ Truong Quoc Vo*b and BoHung Kim (D) *a
}

Heat transfer across an interface between a monolayer coated solid substrate and fluid has been extensively analyzed through a series of non-equilibrium molecular dynamics simulations. The effect of the monolayer was studied by varying its atomic mass $\left(m_{M}\right)$ and interaction energy between monolayer particles $\left(\varepsilon_{\mathrm{MM}}\right)$. Even though the fluid adsorption plays a role in heat transfer at the solid-fluid interface, we found that the interfacial thermal resistance (Kapitza resistance) is highly affected by the insertion of monolayer without any further change in the liquid structure near the solid surface. The Kapitza length monotonically increases with the increase of $m_{M}$ irrespective of $\varepsilon_{M M}$. The observations were explained by analysis of the overlap of the phonon spectrum at the interface using vibrational density of states. The effect of the monolayer on the Kapitza length was summarized by a fourth polynomial function that demonstrates the contribution of both $m_{\mathrm{M}}$ and $\varepsilon_{\mathrm{MM}}$ with respect to each other on the Kapitza resistance within the parametric range studied.

Received 10th October 2018 Accepted 3rd February 2019

DOI: $10.1039 / \mathrm{c} 8 \mathrm{ra0} 0390 \mathrm{~h}$

rsc.li/rsc-advances

Kapitza resistance, such as changing the pressure of the nanoconfined fluid, ${ }^{6,7}$ using different solid lattice orientations, ${ }^{8,9}$ changing the wall temperature, ${ }^{10,11}$ inserting metallic nanoparticles into the fluids (i.e., nanofluids), ${ }^{12,13}$ and nanoengineering the solid substrate. ${ }^{14-17}$ However, wall-fluid interaction energy, which solely determines the surface wettability, ${ }^{18-20}$ is the key factor affecting the Kapitza resistance. Increasing intermolecular interaction strength at the interface (i.e., enhancing the wetting degree) reduces the interfacial thermal resistance and vice versa., ${ }^{3,10,21-27}$ In case of nanoparticle-fluid interfaces, Tascini et al. also showed that large interfacial curvature (nanoparticle size) coupled with strong nanoparticle-fluid interaction strength provide optimum heat transport near the interfacial region. ${ }^{28}$ Moreover, in 2016, Ge et al. used time-domain thermoreflectance to measure the Kapitza length between water and various solids through the transport of thermally excited vibrational energy across the interfaces. ${ }^{29}$ It was reported that the Kapitza length at hydrophobic interfaces $(10-12 \mathrm{~nm})$ is a factor of 2-3 larger than the

$$
L_{\mathrm{K}}=R_{\mathrm{K}} \lambda=\frac{\Delta T}{\left.\frac{\partial T}{\partial z}\right|_{\text {fluid }}}
$$

where $\partial T / \partial z$ is the temperature gradient on the fluid side in the direction ( $z$-axis) of heat transfer.

Recent developments in nanoscience and technology have attracted a vast number of investigations of mass, momentum, and energy transport in nanoscale structures and nanoconduits. ${ }^{4,5}$ Several methods have been used to manipulate the

${ }^{a}$ School of Mechanical Engineering, University of Ulsan, Daehak-ro 93, Namgu, Ulsan 680-749, South Korea. E-mail: bohungk@ulsan.ac.kr

${ }^{b}$ University of Kaiserslautern, Laboratory of Engineering Thermodynamics, ErwinSchrodinger-Str. 44, Kaiserslautern 67663, Germany. E-mail: truong.vo@mv.uni-kl.de

Kapitza length at hydrophilic interfaces (3-6 nm). Nevertheless, changing solid substrate material seems inapplicable, because every device has its own specific application and usage and operating conditions.

For such a case, using two-dimensional (2D) material coated on solid substrate has become a potential solution because one atom thick layers of almost any material are transparent to visible light; however, this can vary the energy transport at the interface. Since the discovery of graphene in $2004,{ }^{30}$ it has become a cutting-edge material; that opens up a pandora's box for other 2D materials that might be beyond the limited current applicability of graphene. Recently, 2D materials have been attracting increasing attention due to the many interesting 
properties originating from bulk to monolayer transition. ${ }^{\mathbf{3 1 , 3 2}}$ For instance, Rafiee et al. measured a 30-40\% increase in condensation heat transfer as a result of the ability of graphene coating to suppress copper oxidation. ${ }^{33}$ The enhancement of condensation heat transfer performance with graphene coatings was also confirmed in the report of Preston et al. ${ }^{34}$ More recently, the results on Kapitza resistance revealed that the interfacial temperature jump increases with the introduction of graphene at the interface between solid and fluid water. ${ }^{35-37}$ Meanwhile, the dominant influence of monolayers $\mathrm{WS}_{2}$ and $\mathrm{MoS}_{2}$ on the wettability of the underlying substrates has also been investigated. ${ }^{38}$ It was shown that even when a monolayer $\mathrm{WS}_{2}$ (or $\mathrm{MoS}_{2}$ ) is coated, the measured contact angle highly increases compared to the substrate without coatings.

For the reasons given above, a systematic study on the effects of monolayer coating on the interfacial heat transfer is required. There have been several studies on thermal boundary resistance at the perfect interfaces between solids, ${ }^{39,40}$ and with the introduction of monolayer near the solid-solid interface. ${ }^{\mathbf{4 1 4 2}}$ However, they lack an investigation on the variation of Kapitza length at solid-fluid interfaces with the presence of monolayer inserted under a wide range of its atomic properties. Note that substrate and monolayer particles are likely to vibrate back and forth in both horizontal and vertical directions about a fixed lattice point unlike the fluid particles, as the interatomic potential is comparatively weak for fluids. In that case, the mutual combination of atomic mass $\left(m_{\mathrm{M}}\right)$ and interaction energy $\left(\varepsilon_{\mathrm{MM}}\right)$ of monolayer may lead to a significant influence in heat transport at the interfacial region. Therefore, in this paper, we aim to study the effects of the interfacial monolayer on Kapitza resistance between solid and fluid using molecular dynamics (MD) simulations by the variation of $m_{\mathrm{M}}$ and $\varepsilon_{\mathrm{MM}}$. Finally, $L_{\mathrm{K}}$ under the effect of monolayer was compared as the function of $m_{\mathrm{M}}$ and $\varepsilon_{\mathrm{MM}}$. It is known that interaction energy $(\varepsilon)$ and mass $(m)$ are related to the thermal oscillation frequency, which is proportional to $\sqrt{\frac{\varepsilon}{\sigma^{2} m}}=\omega \cdot{ }^{22}$ Hence, in this paper, we attempt to correlate $L_{\mathrm{K}}$ and $\omega_{\mathrm{r}}=\frac{\omega_{\mathrm{M}}}{\omega_{\mathrm{F}}}$. Herein, $\omega_{\mathrm{r}}$ denotes the interfacial mismatch at the solid-fluid interface, where $\omega_{\mathbf{M}}$ and $\omega_{\mathrm{F}}$ represents the thermal oscillation frequency of monolayer and fluid particles respectively.

\section{Theoretical background}

The solid wall atoms are initially constructed in perfect FCC structure with a computational domain of $L_{x}=8 a, L_{y}=8 a$ and $L_{z}=15 a$ across each respective direction, where the lattice constant $a=0.5256 \mathrm{~nm}$ (see Fig. 1). Fluid particles were confined between two solid walls with and without monolayer coating. The number density $\left(\rho=N \sigma^{3} / V\right)$ of fluid was set to 0.8 at a distance of $10 \mathrm{~nm}$ along the $z$-direction. For simulations where the interfacial monolayers (colored gray) are presented, the innermost solid layers, (i.e. the ones facing the fluid argon), are replaced by the monolayer. We limit the monolayer to the nonlattice-mismatch to the lattice structure of the solid walls, but with a different atomic mass and interaction energy depending on the cases studied.

To perform the non-equilibrium MD (NEMD) simulations of heat transport through the $z$-direction of the systems, the outermost layers of both sides of the simulation domains (colored cyan) were fixed to their original positions to maintain
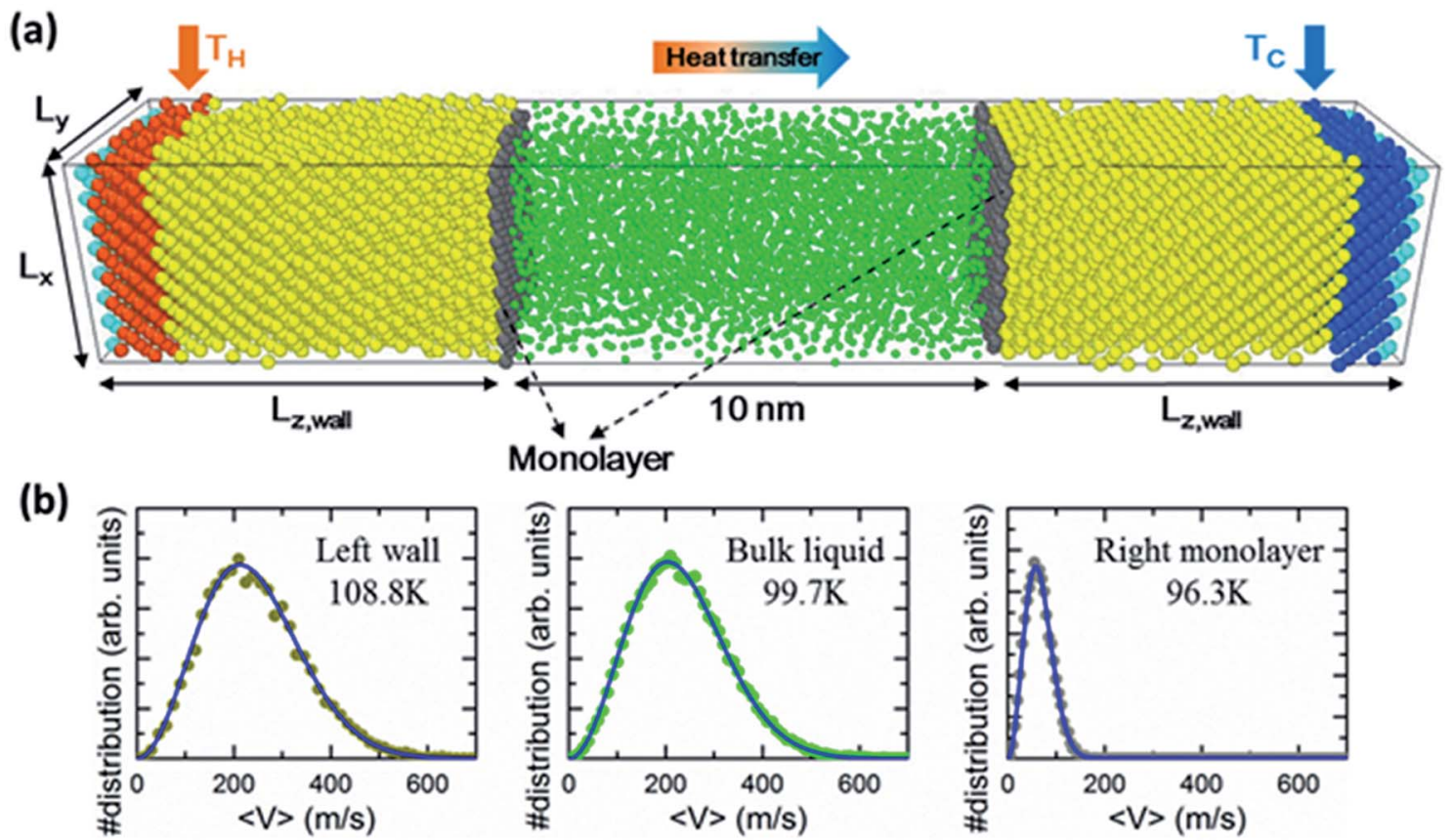

Fig. 1 (a) Snapshot of the MD simulation of heat transfer at the interface between the monolayer-coated solid surface and argon. (b) Velocity distribution for $m_{\mathrm{M}}=12 m_{\mathrm{F}}$ and $\varepsilon_{\mathrm{MM}}=10 \varepsilon_{\mathrm{FF}}$ at the NEMD steady state condition compared to the Maxwell-Boltzmann distribution function at (left) one-atomic-layer in the left wall, (middle) fluid argon in the bulk region, and (right) interfacial monolayer on the right wall. 
a constant volume. Thermostats were situated at four consecutive layers (colored orange and blue) next to the outmost layers. Meanwhile, the remaining solid and fluid atoms were free to move without thermostats applied when heat transfer occurs. The temperature of the left wall was kept higher than the right to generate thermal energy fluxes from left to right. Periodic conditions were applied in the $x$ - and $y$-directions.

In this study, we used the truncated (12-6) Lennard-Jones (LJ) potential to model the interactions between atoms as follows

$$
U_{\text {truncated }}\left(r_{\mathrm{ij}}\right)=4 \varepsilon\left[\left(\left(\frac{\sigma}{r_{\mathrm{ij}}}\right)^{12}-\left(\frac{\sigma}{r_{\mathrm{ij}}}\right)^{6}\right)-\left(\left(\frac{\sigma}{r_{\mathrm{c}}}\right)^{12}-\left(\frac{\sigma}{r_{\mathrm{c}}}\right)^{6}\right)\right],
$$

where $\varepsilon$ is the depth of the potential well, $r_{\mathrm{ij}}$ is the intermolecular distance, $\sigma$ is the finite molecular distance at which the interatomic potential is zero, and $r_{\mathrm{c}}$ is the cut-off distance of $1.0 \mathrm{~nm}$. The interaction parameters and atomic properties used in this study are shown in Table 1 . The interactions between the wall (or substrate) atoms are fixed as $\varepsilon_{\mathrm{WW}}=9 \varepsilon_{\mathrm{FF}}$ and $\sigma_{\mathrm{WW}}=\sigma_{\mathrm{FF}}$. The mass of the wall atoms is fixed as equal to that of fluid argon. The interaction energy between the monolayer atoms $\varepsilon_{\mathrm{MM}}$ is varied between $5 \varepsilon_{\mathrm{FF}}$ and $20 \varepsilon_{\mathrm{FF}}$, whereas $\sigma_{\mathrm{MM}}$ is fixed to $\sigma_{\mathrm{FF}}$. The mass of the monolayer atom is varied between $0.5 m_{\mathrm{F}}$ and $16 m_{\mathrm{F}}$. The intermolecular interaction between the wall and fluid atoms, as well as between the monolayer and fluid atoms are kept $\varepsilon_{\mathrm{WF}}=\varepsilon_{\mathrm{MF}}=0.2 \varepsilon_{\mathrm{FF}}$. Meanwhile, the interaction parameters between the wall and monolayer atoms are estimated from the Lorentz-Berthelot (L-B) mixing rules. ${ }^{43}$ In all simulations, a cut-off distance of $3 \sigma_{\mathrm{FF}}$ is used.

The heat flux $Q$ in the fluid is calculated by using the IrvingKirkwood (I-K) expression as follows: ${ }^{\mathbf{4 4 , 4 5}}$

$$
Q=\frac{1}{\operatorname{vol}}\left\langle\sum_{\mathrm{i}} v_{\mathrm{i}} e_{\mathrm{i}}-\sum_{\mathrm{i}<\mathrm{j}}\left(f_{\mathrm{ij}} v_{\mathrm{j}}\right) r_{\mathrm{ij}}\right\rangle,
$$

where $e_{\mathrm{i}}$ is the per-atom energy (including potential and kinetic energy) of atom $\mathrm{i}, v_{\mathrm{i}}$ is the velocity of atom $\mathrm{i}, f_{\mathrm{ij}}$ is the force acting on atom $\mathrm{i}$ from atom $\mathrm{j}$. Here, the volume was defined within the fluid domain by considering the contribution of each argon molecule.

Once the heat flux is calculated, the thermal conductivity of fluid argon can be obtained from the Fourier law. Also, we compute the vibrational density of states (VDOS) of atoms at the interface using the Fourier transform of its velocity autocorrelation function (VACF) as:

Table 1 Interaction parameters studied in the simulation domain ${ }^{a}$

\begin{tabular}{llll}
\hline Interaction & $\varepsilon$ & $\sigma$ & $m$ \\
\hline Fluid-fluid (F-F) & $\varepsilon_{\mathrm{FF}}$ & $\sigma_{\mathrm{FF}}$ & $m_{\mathrm{F}}$ \\
Wall-wall $(\mathrm{W}-\mathrm{W})$ & $9 \varepsilon_{\mathrm{FF}}$ & $\sigma_{\mathrm{FF}}$ & $m_{\mathrm{F}}$ \\
Monolayer-monolayer (M- & {$[5-20] \varepsilon_{\mathrm{FF}}$} & $\sigma_{\mathrm{FF}}$ & {$[0.5-16] m_{\mathrm{F}}$} \\
$\mathrm{M})$ & & & \\
$\mathrm{M}-\mathrm{W}$ & $\sqrt{\varepsilon_{\mathrm{MM}} \varepsilon_{\mathrm{WW}}}$ & $\left(\sigma_{\mathrm{MM}}+\sigma_{\mathrm{WW}}\right) / 2$ \\
$\mathrm{~W}-\mathrm{F}$ & $0.2 \varepsilon_{\mathrm{FF}}$ & $\sigma_{\mathrm{FF}}$ & \\
$\mathrm{M}-\mathrm{F}$ & $0.2 \varepsilon_{\mathrm{FF}}$ & $\sigma_{\mathrm{FF}}$ & \\
${ }^{a} \varepsilon_{\mathrm{FF}}=0.0103 \mathrm{eV} . \sigma_{\mathrm{FF}}=0.3405 \mathrm{~nm} . m_{\mathrm{F}}=39.948$ gram per mol.
\end{tabular}

$$
\operatorname{VDOS}(\omega)=\int_{0}^{+\infty} Z(t) \mathrm{e}^{-\mathrm{i} \omega t} \mathrm{~d} t
$$

where the VACF is defined as $Z(t)=\langle\vec{v}(t) \cdot \vec{v}(0)\rangle$. According to condensed matter physics, the VDOS of a system characterized by the number of states per interval of energy at each energy level that is available to be occupied by phonons.

Simulations start with an NVT (i.e., constant number of atoms, constant volume, and constant temperature) ensemble applied to the entire system. In this stage, the Maxwell-Boltzmann velocity distribution is used for the initial velocities of all atoms, while the Nose-Hoover thermal thermostat maintains system temperature at $100 \mathrm{~K}$. Subsequently, the hot and cold reservoirs are respectively subjected to $110 \mathrm{~K}$ and $90 \mathrm{~K}$ using Langevin thermostats NVE (i.e., constant number of atoms, constant volume, and constant energy). The simulation time step is set to $1.0 \mathrm{fs}$. The simulations are performed for $12 \mathrm{~ns}$ : the first $3 \mathrm{~ns}$ to allow the systems to reach equilibrium, the next $3 \mathrm{~ns}$ to ensure the systems to gain a steady state in the presence of heat flux, and the last 6 ns for averaging. All simulations in this study are carried out using LAMMPS. ${ }^{46}$

Before we present the temperature distributions and discuss the effects of the monolayer, we must demonstrate the local thermal equilibrium (LTE) in the system. It is known that the local temperature can be defined only if the LTE is established. In this paper, the temperature distributions are obtained using slab bins parallel to the walls. For instance, slab bins $0.2628 \mathrm{~nm}$ and $0.4 \mathrm{~nm}$ in thickness are used to observe the local temperatures in solid walls and fluids, respectively. Fig. 1b shows the distribution of velocity components of atoms contained in one bin at different positions (i.e., in the mean position of the left solid wall, in the mean position of the fluid region, and at the mean position of the monolayer particles in the colder side when $m_{\mathrm{M}}=12 m_{\mathrm{F}}$ and $\left.\varepsilon_{\mathrm{MM}}=10 \varepsilon_{\mathrm{FF}}\right)$. The results reveal that atomic velocity components satisfy the Maxwell-Boltzmann distribution very well; thus, the LTE is established.

\section{Results and discussion}

\subsection{Effect of monolayer on interfacial heat transfer}

To provide a general picture of the effect of monolayer coating on heat transfer at solid-fluid interfaces, we show in Fig. 2 the temperature distributions along the $z$-direction of channels without and with monolayer. From Fig. 2b-d, we keep the interaction energy of monolayer at $\varepsilon_{\mathrm{MM}}=10 \varepsilon_{\mathrm{FF}}$, while gradually increasing its mass. In order to determine the interfacial temperature jumps, we extrapolate the linear temperature from the fluid region to the solid surfaces, which are described by the black arrows. Throughout our analysis, surface positions are defined at the mean position of the solid surface adjacent to the fluid. When the monolayer is coated, the surface positions are then at the mean position of the monolayers. We also observe additional temperature jumps between the solid substrate and monolayer interfaces, indicating the impact of solid-solid interactions of nanocomposite structure into the overall thermal resistance at solid-fluid interface. For such a case, the temperature jumps need to be defined considering both 
(a) Without Monolayer

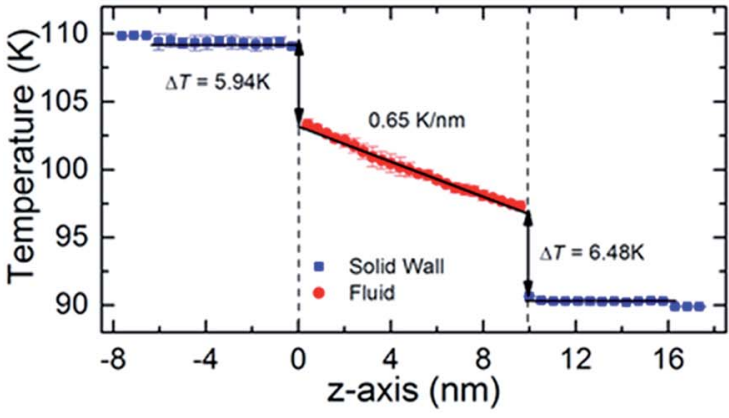

(c) $\varepsilon_{\mathrm{um}}=10 \varepsilon_{\mathrm{F}}, m_{\mathrm{u}}=10 \mathrm{~m}_{\mathrm{F}}, \omega_{\mathrm{u}}=\omega_{\mathrm{s}}$

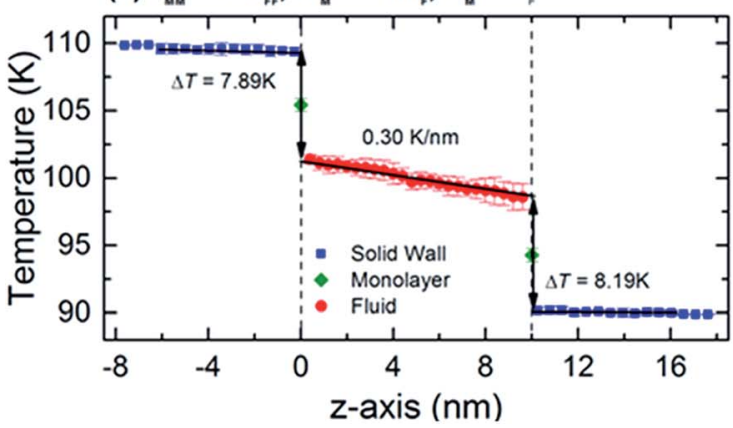

(b) $\varepsilon_{\mathrm{uu}}=10 \varepsilon_{\mathrm{F}}, m_{\mathrm{M}}=0.5 m_{\mathrm{F}}, \omega_{\mathrm{M}}=4.47 \omega_{\mathrm{F}}$

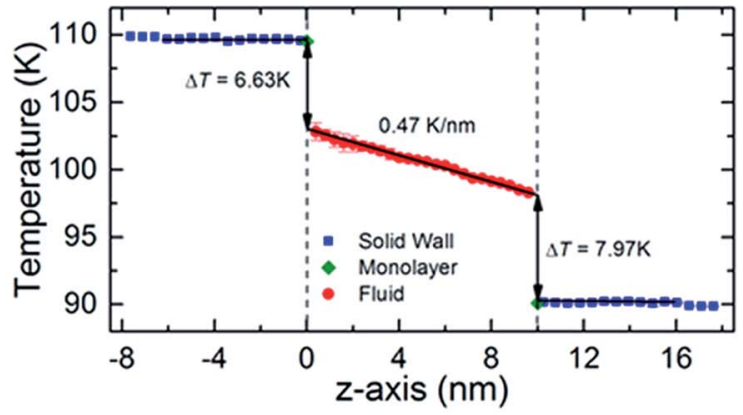

(d) $\varepsilon_{M M}=10 \varepsilon_{F F}, m_{M}=16 m_{F}, \omega_{M}=0.79 \omega_{F}$

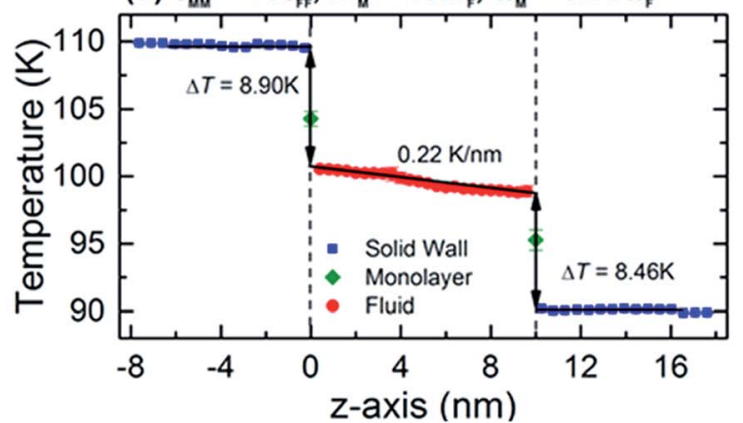

Fig. 2 Typical temperature profiles across the wall-fluid interface (a) without and (b-d) with monolayer coated for $\varepsilon_{\mathrm{MM}}=10 \varepsilon_{\mathrm{FF}}$ at various values of $m_{\mathrm{M}}$.

interface (i.e., monolayer-fluid) and near-interface (wallmonolayer) temperature discontinuity. Therefore, we extrapolate the linear temperature from the wall substrate across the monolayer. The results shown in Fig. 2 demonstrate the effects of monolayer as seen in the variation in temperature jump and temperature gradient within the fluid region compared to the substrate-fluid case. In addition, there is a slight discrepancy in temperature jumps at the hot and cold sides; however, no tendency is observed. Therefore, in the following, Kapitza length is shown as an average of the two walls.

Fig. 3 shows the thermal conductivity $(\lambda)$ measured in the confined fluid. The average thermal conductivity of the bulk

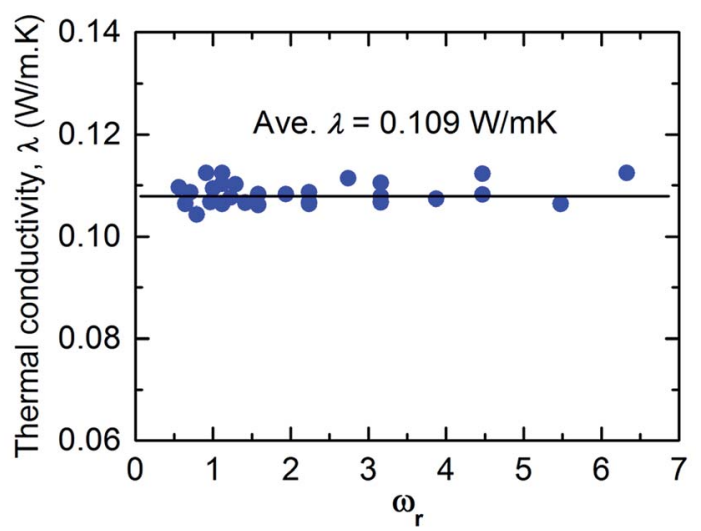

Fig. 3 Dependence of thermal oscillation frequency between monolayer and fluid on thermal conductivity (black line represents the value for $\lambda$ without monolayer coating). fluid Argon at $100 \mathrm{~K}$ is in agreement with other MD studies, (where the relative deviation of $\lambda$ is $0.9 \%$ to $7.66 \%$ ), as well as in experiments $^{\mathbf{4 8 , 4 9}}$ (where the relative deviation of $\lambda$ is $3.2 \%$ to $4.63 \%)$. The results from Fig. 3 reveal that the thermal resistance on the interface solely depends on the interfacial characteristics, and the thermal conductivity of the fluid is independent of the thermal resistance at the interface. In this study, the effect of monolayer is studied by the variation of its atomic mass $\left(m_{\mathrm{M}}\right)$ and particle-particle interaction energy $\left(\varepsilon_{\mathrm{MM}}\right)$. Therefore, it is interesting to know the contribution of $m_{\mathrm{M}}$ and $\varepsilon_{\mathrm{MM}}$ to the interfacial heat transfer.

\subsection{Role of monolayer mass and interaction energy}

3.2.1 Impact on Kapitza length. At first, we define $L_{\mathrm{K}, \mathrm{r}}$ as the ratio of the Kapitza length with a monolayer $\left(L_{\mathrm{K}-\mathrm{WMF}}\right)$ to one without a monolayer coating $\left(L_{\mathrm{K}-\mathrm{WF}}\right)$ near the wall-fluid interfacial region $\left(\right.$ i.e., $\left.L_{\mathrm{K}, \mathrm{r}}=\frac{L_{\mathrm{K}-\mathrm{WMF}}}{L_{\mathrm{K}-\mathrm{WF}}}\right)$. Therefore, we plot the variation of Kapitza length versus monolayer mass (c.f. Fig. 4a) and monolayer interaction energy (c.f. Fig. $4 \mathrm{~b}$ ). Here, after reaching the minimum, $L_{\mathrm{K}, \mathrm{r}}$ increases monotonically with the increase of the monolayer mass independent of its interaction energy. In addition, the monolayer surface for different $\varepsilon_{\mathrm{MM}}$ doesn't show any significant effect on $L_{\mathrm{K}, \mathrm{r}}$. Hence it would seem to be the monolayer-mass that plays a dominant role in the interfacial thermal resistance, as demonstrated also in Fig. 2.

Note that the vibrating period of monolayer particles continuously gets longer with subsequent increase of $m_{\mathbf{M}}$ regardless of any lower value for $\varepsilon_{\mathrm{MM}}$. Thus, the monolayer 
(a)

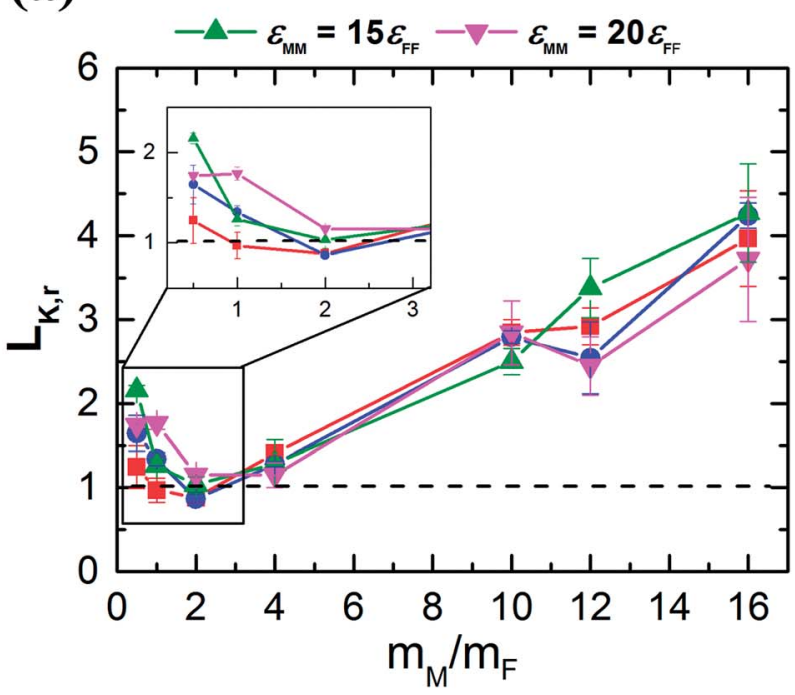

(b)

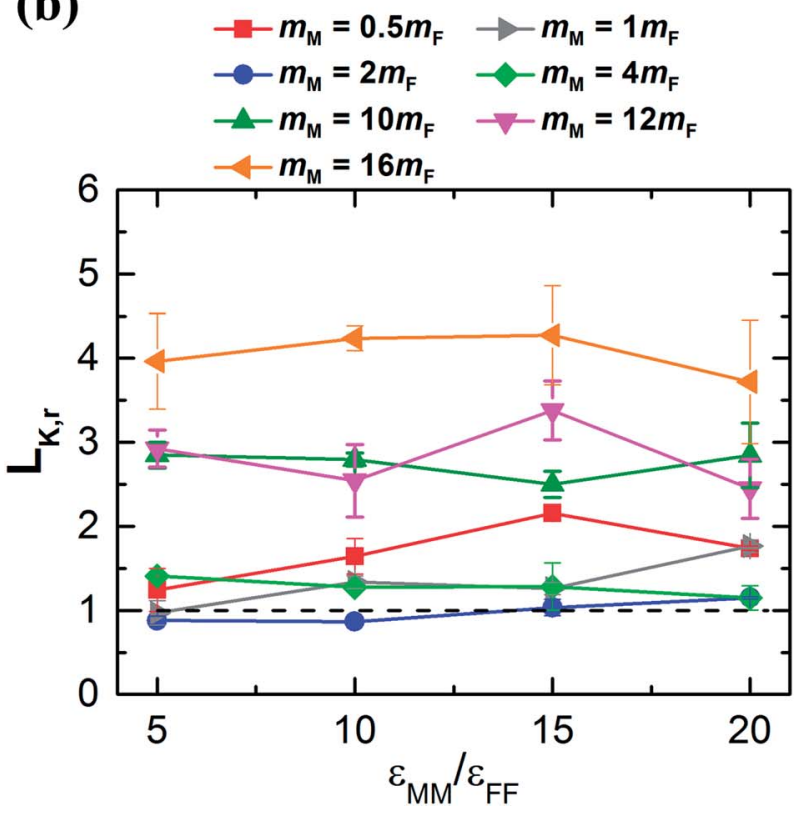

Fig. 4 Variation of $L_{K, r}$ as a function of (a) $m_{M}$ at different $\varepsilon_{M M} / \varepsilon_{F F}$ and (b) $\varepsilon_{\mathrm{MM}} / \varepsilon_{\mathrm{FF}}$ at different $m_{\mathrm{M}}$.

surface continuously becomes very rough. However, the amplitude of the respective particles weakly depends on atomic mass for a high stiff surface (increased interaction energy between surface particles) that is largely controlled by the wall's temperature. ${ }^{50-52}$ Primarily, $L_{\mathrm{K}, \mathrm{r}}$ is found to be smaller for lighter monolayer particles than heavier particles for all the cases of $\varepsilon_{\mathrm{MM}}$ (see Fig. 4a). Starting from $m_{\mathrm{M}}=0.5 m_{\mathrm{F}}, L_{\mathrm{K}, \mathrm{r}}$ likely to decrease mainly due to the reduced vibrational mismatch between the absorbed fluid and the solid surface at the interfacial region. Consequently, a reduced $L_{\mathrm{K}, \mathrm{r}}$ is observed for a delicate amount of mass at $m_{\mathrm{M}}=2 m_{\mathrm{F}}$, which marginally varies for different $\varepsilon_{\mathrm{MM}}$ around the dashed line. This line represents the result of Kapitza length at the solid-fluid interface without monolayer coating. Afterward the interfacial region experienced a greater vibrational mismatch between the respective materials, and eventually, the $L_{\mathrm{K}, \mathrm{r}}$ becomes critical for heavy monolayer particles (i.e., $m_{\mathrm{M}}>2 m_{\mathrm{F}}$ ). These results indicate that the thermal vibration of monolayer surface, which is light in weight, similarly correlated with the wall and fluid particles as the vibrating period is considerably very short. In contrary, heavier monolayer molecules further hinder the heat transport due to the increased elastic properties as well as long vibrating period at the substrate-monolayer and monolayer-fluid region in spite of any alteration of $\varepsilon_{\mathrm{MM}}$. However, it is still unclear what factor mainly contributes to the $L_{\mathrm{K}, \mathrm{r}}$ for different values of $\varepsilon_{\mathrm{MM}}$.

In Fig. $4 \mathrm{~b}$, the $L_{\mathrm{K}, \mathrm{r}}$ fluctuates around an average value along each respective mass curve; therefore, variation in $\varepsilon_{\mathrm{MM}}$ contributes a minor part to further change in the $L_{\mathrm{K}, \mathrm{r}}$. For the case of $m_{\mathrm{M}}=2 m_{\mathrm{F}}, L_{\mathrm{K}, \mathrm{r}}$ is slightly under the dashed line when $\varepsilon_{\mathrm{MM}}=5 \varepsilon_{\mathrm{FF}}$, that has gone up to more than unity when $\varepsilon_{\mathrm{MM}}=$ $20 \varepsilon_{\mathrm{FF}}$. Such an observation also has been found for almost the entire remaining strong monolayer surface $\left(\varepsilon_{\mathrm{MM}}=15 \varepsilon_{\mathrm{FF}}\right.$ or $\left.20 \varepsilon_{\mathrm{FF}}\right)$. This indicates that $\varepsilon_{\mathrm{MM}}$ has an indirect but important effect at the interface when the substrate is coated with monolayer. In 2011, Liang and Tsai studied the effect of single atom thick film confined between two dissimilar solids. ${ }^{41}$ They also found that the interfacial thermal resistance slightly increases with the increase of film-solid binding strength.

3.2.2 Impact on density profiles. To provide more insight into the effects of monolayer mass and monolayer interaction energy, we first explore the distribution of fluid argon near the surface as shown in Fig. 5. For all cases, fluid atoms form
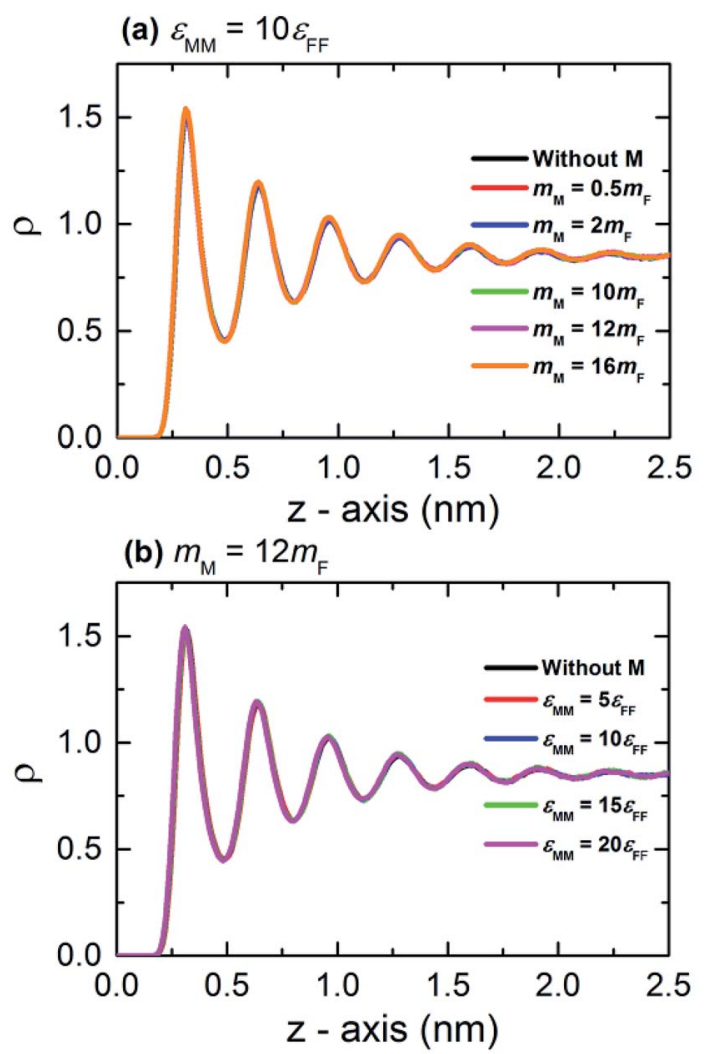

Fig. 5 Density profiles near the hot wall for (a) $\varepsilon_{\mathrm{MM}}=10 \varepsilon_{\mathrm{FF}}$ at various values of $m_{\mathrm{M}}$ and for (b) $m_{\mathrm{M}}=12 m_{\mathrm{F}}$ at various values of $\varepsilon_{\mathrm{FF}}$. 
layered structures due to surface force penetration depth and local fluid-fluid interactions. The molecular structure of fluid at the interface is an important factor in understanding the interfacial thermal resistance. During recent decades, density peaks and depletion length (i.e., the distance between the solid surface and first peak density) have been two major factors for explaining the energy and momentum transport at the interface. ${ }^{7,10,21,35,53,54}$ However, the results from Fig. 5 show that the fluid spatial density distributions remain unchanged in spite of any change in monolayer properties. For instance, the first peak of number density near the solid surface as a function of $m_{\mathrm{M}}$ and $\varepsilon_{\mathrm{MM}}$ is $1.5317 \pm 0.0097$ and $1.5314 \pm 0.0078$ respectively for all of the cases. These observations indicate that the fluid argon was unable to change their motion after interacting with the monolayer particles. This dearth of concurrence between fluid and monolayer coated solid particles leads to the more discontinuity at the interface, which results in higher interfacial thermal resistance. Besides, change in $\varepsilon_{\mathrm{MM}}$ has no impact on the interaction between monolayer and fluid particles. This tendency suggests that the thermal oscillation frequency of monolayer particles doesn't have any effect on the fluid layering near the solid-fluid interface. However, Asproulis et al. Frank et al. found that the fluid density near the surface is highly affected by the variation of wall mass and stiffness, which contrast to our results..$^{51,52,55}$ The reason is that, in their case, the elastic properties of all the wall particles were varied to quantify the oscillatory motion of fluid particles. Therefore, both the roughness and smoothness of solid surface play a dominant role on the fluid structure near the interface.
3.2.3 Impact on vibrational density of states. To comprehensively understand the influence of monolayer coating on $L_{\mathrm{K}, \mathrm{r}}$, we investigate the vibrational density of states, (VDOS; i.e., number of vibrational modes per unit volume and frequency), during NEMD simulation using eqn (4). Particularly VDOS helps to quantify the change of phonon spectrum due to the mismatch in vibrational properties of different crystal structures in contact across an interface. ${ }^{56}$ Besides, at nanoscale, thermal motions of fluid particles near the solid surface started to freeze, and form a layered structure at the solid-fluid interface due to the surface force and local fluid-fluid interaction. This layer also referred to as the "solid-like fluid layer". ${ }^{10,21,50,53}$ Therefore, we consider the first fluid layered structure near the solid surface to calculate the VDOS of fluid particles. In Fig. 6, the overlap portion of the phonon spectrum is quite large for the interface with lighter monolayer atoms, including only the substrate-fluid interface, than heavier monolayer atoms when $\varepsilon_{\mathrm{MM}}=10 \varepsilon_{\mathrm{FF}}$. Basically, the overlap degree at the interface measures the degree of interfacial vibrational coupling. Increasing vibrational coupling at the interface enhances the thermal transport and thus reduces the thermal resistance. Hence, the crystal structure having $m_{\mathrm{M}}=$ $0.5 m_{\mathrm{F}}, m_{\mathrm{M}}=2 m_{\mathrm{F}}$ and without monolayer interface experienced relatively much lower ITR than the interface with $m_{\mathrm{M}}=4 m_{\mathrm{F}}, m_{\mathrm{M}}$ $=10 m_{\mathrm{F}}$ and $m_{\mathrm{M}}=16 m_{\mathrm{F}}$, which further corroborates the details in Fig. 4. However, almost the entire phonon spectrum due to the monolayer has been used in the overlap region when $m_{\mathrm{M}}=2 m_{\mathrm{F}}$. In that case, peak of the phonon spectrum of the monolayer is also in good agreement with both peaks of the innermost substrate layer and the remaining bulk substrate layers, indicating a better vibrational coupling than any other cases of VDOS.

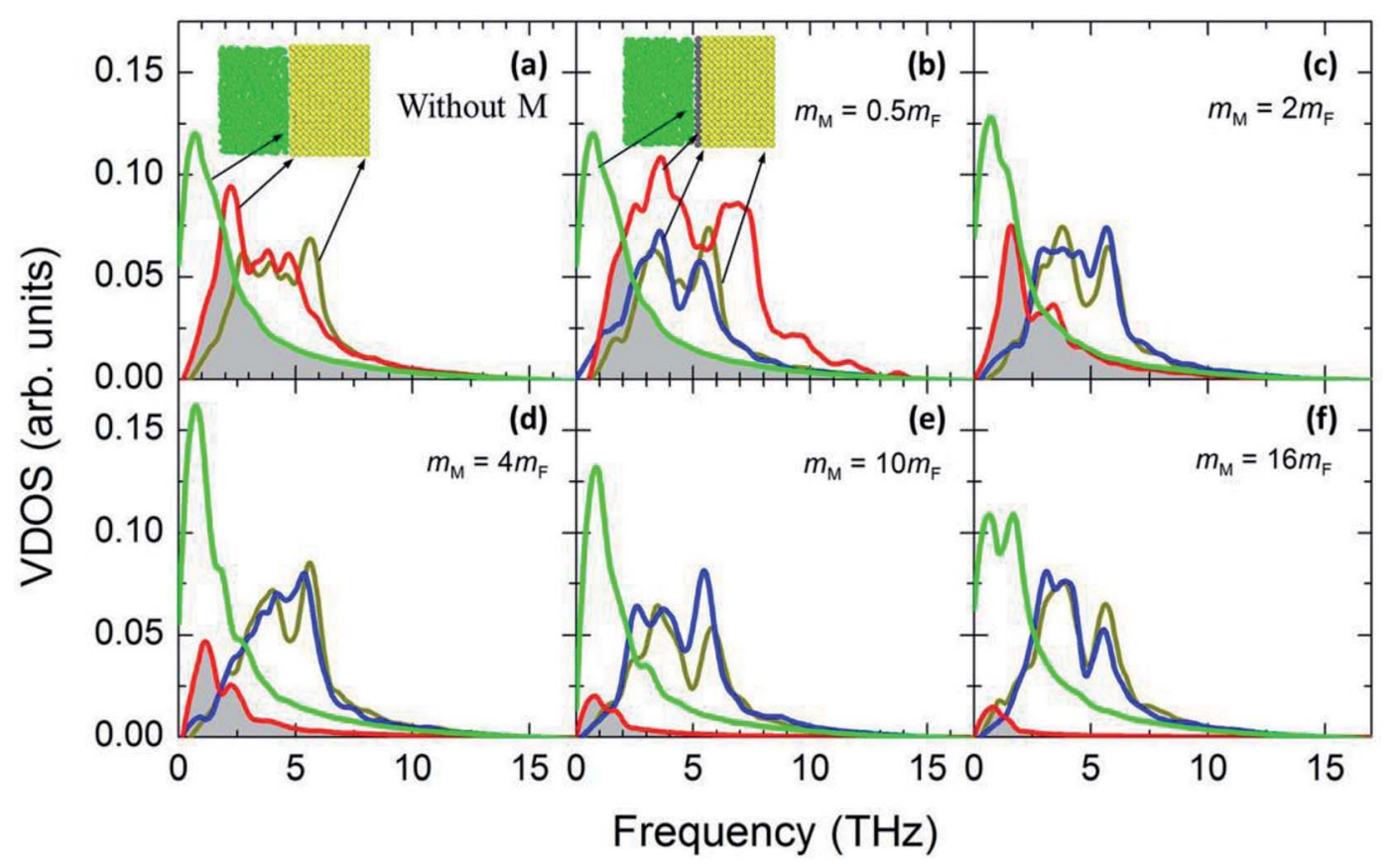

Fig. 6 VDOS of wall, monolayer and fluid argon atoms as a function of frequency for $\varepsilon_{\mathrm{MM}}=10 \varepsilon_{\mathrm{FF}}$ at different values of $m_{\mathrm{M}}$. (a) Interface without monolayer coating: surface atoms in fluid (green line), innermost substrate (red line) and bulk substrate (brown line). (b)-(f) Interface with monolayer coating: surface atoms in fluid (green line), monolayer (red line), innermost substrate (blue line) and bulk substrate (brown line). The grey area illustrates the overlap between VDOS. 
Fig. 7 illustrates the possible scenario of the VDOS with the variation of $\varepsilon_{\mathrm{MM}}$ for $m_{\mathrm{M}}=10 \mathrm{~m}_{\mathrm{F}}$. Here the black line represents the innermost solid layer, and the colored lines represent the monolayer particles. Apparently, there is no obvious change in the phonon states of the monolayer with the increase of $\varepsilon_{\mathrm{MM}}$, and the difference in peaks between the innermost solid layer and monolayer is considerably large. This indicates that $\varepsilon_{\mathrm{MM}}$ has no such control over the thermal energy transport at the interfacial region for the heavy monolayer particles. Liang and Tsai also studied the VDOS of a thin film between two different solid materials. ${ }^{41}$ They found better vibrational coupling at the solid-film-solid interface with the increase of film-solid binding strength. Since the solid particles oscillate about a fixed lattice point unlike fluids; therefore, increased inelastic or strong solid surface was likely to have a good agreement in the vibrations with the thin film near the interface.

3.2.4 Effect of thermal oscillation frequency on the Kapitza length. In Fig. 8, the results of the numerical experiments are summarized to take into account the overall effects of monolayer mass and interaction energy on Kapitza length at the interface between fluid argon and the solid substrate as a function of the thermal oscillation frequency. To quantify the behavior, we introduce a fourth-order polynomial master curve.

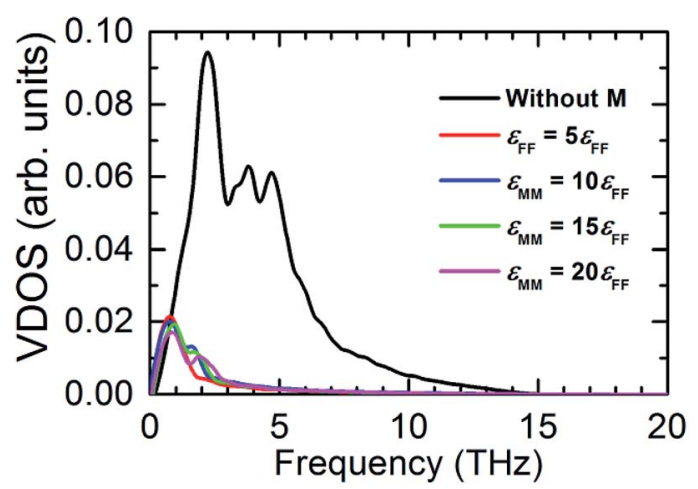

Fig. 7 VDOS of innermost wall and monolayer atoms for $m_{\mathrm{M}}=10 m_{\mathrm{F}}$ at different values of $\varepsilon_{\mathrm{MM}}$.

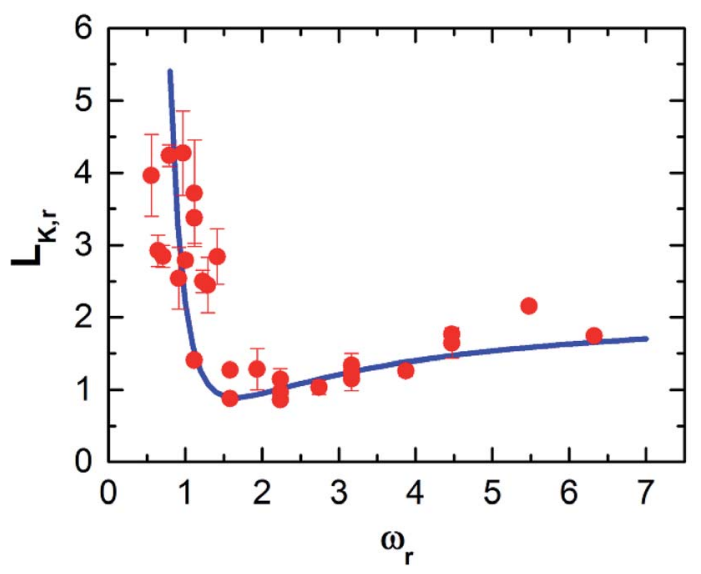

Fig. 8 "Master" curve describing the variation of the Kapitza length at the interface between fluid argon and monolayer-coated solid substrate as a function of oscillating frequency.

$$
L_{\mathrm{K}, \mathrm{r}}=a\left(\frac{1}{\omega_{\mathrm{r}}{ }^{4}}-\frac{1}{{\omega_{\mathrm{r}}}^{3}}+\frac{1}{{\omega_{\mathrm{r}}{ }^{2}}^{2}}-\frac{1}{\omega_{\mathrm{r}}{ }^{1}}\right)+b
$$

in which values of the coefficients $a=4$ and $b=2.2$ are obtained through data fitting. For all the examined cases in the current study, $L_{\mathrm{K}, \mathrm{r}}$ began to decrease sharply with increasing $\omega_{\mathrm{r}}$ and reached a minimum value within a very low $\omega_{\mathrm{r}}$ region. Afterward $L_{\mathrm{K}, \mathrm{r}}$ was continuing through a marginal upward phase to finally converge to a pulsating value within the parametric range in this study. It is obvious that higher values of $m_{\mathrm{M}}$ will eventually be in a lower-frequency region independent of $\varepsilon_{\mathrm{MM}}$. In that case, $L_{\mathrm{K}, \mathrm{r}}$ is found to be very high for predominantly heavier monolayer particles. Therefore, when the monolayer surface becomes heavier, there will be a significant variation in the thermal vibration of the substrate and fluid particles that further disturbs the heat transport across the interfacial region regardless of $\varepsilon_{\mathrm{MM}}$. This observation further justifies the details obtained from Fig. 7. In contrast, higher values of $\varepsilon_{M M}$ will surely be in the higher-frequency region and lower values of $\varepsilon_{\mathrm{MM}}$ in the lower-frequency region, regardless of a small amount of $m_{\mathrm{M}}$. As a result, the interface experienced lower thermal resistance than the large amount of $m_{\mathrm{M}}$. Hence, lighter monolayer molecules for any kind of $\varepsilon_{\mathrm{MM}}$ tend to be consistent with the thermal vibration between substrate and fluid particles, which helps to minimize the deficit of heat transport across the interfacial region. Despite having a better vibrational coupling for lighter $m_{\mathrm{M}}$, after reaching a minimum value, $L_{\mathrm{K}, \mathrm{r}}$ increases gradually with increasing $\omega_{\mathrm{r}}$ as well as $\varepsilon_{\mathrm{MM}}$. This indicates that the strong monolayer surface created more $L_{\mathrm{K}, \mathrm{r}}$ than a flexible monolayer surface for lighter monolayer particles. Hence, $\varepsilon_{\mathrm{MM}}$ act as a deciding factor for the interfacial thermal resistance when the $m_{\mathrm{M}}$ is likely to have a low weight. In 2008, Kim et al. also suggest that Kapitza length increases with the increase of thermal oscillation frequency, which is in accordance with the lighter monolayer particles. ${ }^{3}$ Therefore, a minimum is appeared in the master curve to signify the effect of $m_{\mathrm{M}}$ and $\varepsilon_{\mathrm{MM}}$ as a function of thermal oscillation frequency.

\section{Conclusions}

The role of monolayer particles mass $\left(m_{\mathrm{M}}\right)$ and interaction energy $\left(\varepsilon_{\mathrm{MM}}\right)$ on Kapitza length between the monolayer coated solid surface and fluid argon has been extensively analyzed through non-equilibrium molecular dynamics simulation. Within this monoatomic nano-composite structure, thermal conductivity of fluid showed a constant value at $100 \mathrm{~K}$ using Fourier's law. The fluid density structure near the solid surface is also independent of any change in $m_{\mathbf{M}}$ and $\varepsilon_{\mathbf{M M}}$. This indicates that heat transport at the interfacial region solely depends on the monolayer properties. Considering the mutual combination of $m_{\mathrm{M}}$ and $\varepsilon_{\mathrm{MM}}$, we also observed that the temperature jump increases with decreasing $\omega_{\mathrm{r}}$, suggesting a greater interfacial mismatch between the particles with a lower oscillation frequency.

Primarily, our results indicate that $m_{\mathbf{M}}$ is the dominant factor for controlling the amount of heat transport at the interface as the Kapitza length monotonically increases with increasing $m_{\mathrm{M}}$, 
regardless of $\varepsilon_{\mathrm{MM}}$. Because the vibrational mismatch of atoms continuously increases with the subsequent addition of mass, the measured $L_{\mathrm{K}, \mathrm{r}}$ was expectedly much higher for heavier monolayer particles than lighter monolayer particles for a given value of $\varepsilon_{\mathrm{MM}}$. This is because the interaction energy between heavier monolayer particles has no control over the interfacial thermal resistance which results in a greater vibrational mismatch between the materials near the interface. On the other hand, for lighter monolayer particles, the interaction energy of monolayers is likely to maintain a better vibrational coupling with the solid substrate. Therefore, even if the vibrational mismatch marginally increases with the increase of $m_{\mathrm{M}}$, $\varepsilon_{\mathrm{MM}}$ minimizes that mismatch which eventually leads to a reduced Kapitza resistance at the interfacial region.

Further improvement of interfacial thermal transport is possible when the nano-composite materials remain mismatched in the lattice structure. Hence, further examinations of the lattice mismatch monolayer surface with the existing monatomic crystal structure will be a part of our future research.

\section{Conflicts of interest}

There are no conflicts to declare.

\section{Acknowledgements}

This work was supported by the 2018 Research Fund of University of Ulsan.

\section{References}

1 P. L. Kapitza, J. Phys., 1941, 4, 181.

2 P. A. Thompson and S. M. Troian, Nature, 1997, 389, 360362.

3 B. H. Kim, A. Beskok and T. Cagin, J. Chem. Phys., 2008, 129, 174701.

4 G. E. Karniadakis, A. Beskok and N. Aluru, Microflows and Nanoflows: Fundamentals and Simulation, Springer-Verlag, New York, 2005.

5 D. G. Cahill, P. V. Braun, G. Chen, D. R. Clarke, S. Fan, K. E. Goodson, P. Keblinski, W. P. King, G. D. Mahan, A. Majumdar, H. J. Maris, S. R. Phillpot, E. Pop and L. Shi, Appl. Phys. Rev., 2014, 1, 011305.

6 H. Han, S. Mérabia and F. Müller-Plathe, J. Phys. Chem. Lett., 2017, 8, 1946-1951.

7 A. Pham, M. Barisik and B. Kim, J. Chem. Phys., 2013, 139, 244702.

8 T. Ohara and D. Torii, J. Chem. Phys., 2005, 122, 214717.

9 A. R. bin Saleman, H. K. Chilukoti, G. Kikugawa, M. Shibahara and T. Ohara, Int. J. Heat Mass Transfer, 2017, 105, 168-179.

10 M. Barisik and A. Beskok, Int. J. Therm. Sci., 2014, 77, 47-54.

11 Z. Shi, M. Barisik and A. Beskok, Int. J. Therm. Sci., 2012, 59, 29-37.

12 H. Han, S. Merabia and F. Müller-Plathe, Nanoscale, 2017, 9, 8314-8320.
13 P. Keblinski, S. R. Phillpot, S. U. S. Choi and J. A. Eastman, Int. J. Heat Mass Transfer, 2002, 45, 855-863.

14 H. Hu and Y. Sun, J. Appl. Phys., 2012, 112, 053508.

15 Y. Wang and P. Keblinski, Appl. Phys. Lett., 2011, 99, 073112.

16 K. M. Issa and A. A. Mohamad, Phys. Rev. E: Stat., Nonlinear, Soft Matter Phys., 2012, 85, 031602.

17 T. Q. Vo and B. Kim, J. Chem. Phys., 2018, 148, 034703.

18 T. Q. Vo, M. Barisik and B. Kim, Phys. Rev. E: Stat., Nonlinear, Soft Matter Phys., 2015, 92, 053009.

19 S. Becker, H. M. Urbassek, M. Horsch and H. Hasse, Langmuir, 2014, 30, 13606-13614.

20 M. Barisik and A. Beskok, Mol. Simul., 2013, 39, 700-709.

21 T. Q. Vo and B. Kim, Int. J. Precis. Eng. Manuf., 2015, 16, 1341-1346.

22 B. Kim, Chem. Phys. Lett., 2012, 554, 77-81.

23 J.-L. Barrat and F. Chiaruttini, Mol. Phys., 2003, 101, 16051610.

24 J. Ghorbanian and A. Beskok, Phys. Chem. Chem. Phys., 2017, 19, 10317-10325.

25 J. Ghorbanian, A. T. Celebi and A. Beskok, J. Chem. Phys., 2016, 145, 184109.

26 T. Q. Vo, B. Park, C. Park and B. Kim, J. Mech. Sci. Technol., 2015, 29, 1681-1688.

27 B. H. Kim, A. Beskok and T. Cagin, Microfluid. Nanofluid., 2010, 9, 31-40.

28 A. S. Tascini, J. Armstrong, E. Chiavazzo, M. Fasano, P. Asinari and F. Bresme, Phys. Chem. Chem. Phys., 2017, 19, 3244-3253.

29 Z. Ge, D. G. Cahill and P. V. Braun, Phys. Rev. Lett., 2006, 96, 186101.

30 K. S. Novoselov, A. K. Geim, S. V. Morozov, D. Jiang, Y. Zhang, S. V. Dubonos, I. V. Grigorieva and A. A. Firsov, Science, 2004, 306, 666-669.

31 R. Mas-Ballesté, C. Gómez-Navarro, J. Gómez-Herrero and F. Zamora, Nanoscale, 2011, 3, 20-30.

32 D. Akinwande, C. J. Brennan, J. S. Bunch, P. Egberts, J. R. Felts, H. Gao, R. Huang, J.-S. Kim, T. Li, Y. Li, K. M. Liechti, N. Lu, H. S. Park, E. J. Reed, P. Wang, B. I. Yakobson, T. Zhang, Y.-W. Zhang, Y. Zhou and Y. Zhu, Extreme Mech. Lett., 2017, 13, 42-77.

33 J. Rafiee, X. Mi, H. Gullapalli, A. V. Thomas, F. Yavari, Y. Shi, P. M. Ajayan and N. A. Koratkar, Nat. Mater., 2012, 11, 217222.

34 D. J. Preston, D. L. Mafra, N. Miljkovic, J. Kong and E. N. Wang, Nano Lett., 2015, 15, 2902-2909.

35 B. Ramos-Alvarado, S. Kumar and G. P. Peterson, J. Phys. Chem. Lett., 2016, 7, 3497-3501.

36 A. T. Pham, M. Barisik and B. Kim, Int. J. Heat Mass Transfer, 2016, 97, 422-431.

37 T. Q. Vo and B. Kim, Sci. Rep., 2016, 6, 33881.

38 P. K. Chow, E. Singh, B. C. Viana, J. Gao, J. Luo, J. Li, Z. Lin, A. L. Elías, Y. Shi, Z. Wang, M. Terrones and N. Koratkar, ACS Nano, 2015, 9, 3023-3031.

39 R. J. Stevens, L. V. Zhigilei and P. M. Norris, Int. J. Heat Mass Transfer, 2007, 50, 3977-3989.

40 S. Merabia and K. Termentzidis, Phys. Rev. B: Condens. Matter Mater. Phys., 2012, 86, 094303. 
41 Z. Liang and H.-L. Tsai, J. Phys.: Condens. Matter, 2011, 23, 495303.

42 Z. Liang and H.-L. Tsai, Int. J. Heat Mass Transfer, 2012, 55, 2999-3007.

43 M. P. Allen and D. J. Tildesley, Computer Simulation of Liquids, Oxford University Press, 2nd edn, 2017.

44 J. H. Irving and J. G. Kirkwood, J. Chem. Phys., 1950, 18, 817829.

45 B. D. Todd, P. J. Daivis and D. J. Evans, Phys. Rev. E: Stat. Phys., Plasmas, Fluids, Relat. Interdiscip. Top., 1995, 51, 4362-4368.

46 S. Plimpton, J. Comput. Phys., 1995, 117, 1-19.

47 A. J. H. McGaughey and M. Kaviany, Int. J. Heat Mass Transfer, 2004, 47, 1783-1798.

48 B. J. Bailey and K. Kellner, Physica, 1968, 39, 444-462.
49 H. Ziebland and J. T. A. Burton, Br. J. Appl. Phys., 1958, 9, 52. 50 B. H. Kim, A. Beskok and T. Cagin, Microfluid. Nanofluid., 2008, 5, 551-559.

51 N. Asproulis and D. Drikakis, Phys. Rev. E: Stat., Nonlinear, Soft Matter Phys., 2011, 84, 031504.

52 N. Asproulis and D. Drikakis, Phys. Rev. E: Stat., Nonlinear, Soft Matter Phys., 2010, 81, 061503.

53 L. Xue, P. Keblinski, S. R. Phillpot, S. U.-S. Choi and J. A. Eastman, Int. J. Heat Mass Transfer, 2004, 47, 4277-4284.

54 A. T. Pham, M. Barisik and B. Kim, Int. J. Precis. Eng. Manuf., 2014, 15, 323-329.

55 M. Frank, M. Kio, D. Drikakis, L. Könözsy and N. Asproulis, J. Comput. Theor. Nanosci., 2018, 15, 141-146.

56 E. T. Swartz and R. O. Pohl, Rev. Mod. Phys., 1989, 61, 605668. 